\title{
EL INCREMENTO DEL AUTOCONOCIMIENTO Y LA TOMA DE DECISIONES EN ALUMNOS DE EDUCACIÓN SECUNDARIA OBLIGATORIA, DE CUENCA
}

\author{
THE INCREASE OF SELF-KNOWLEDGE AND DECISION-TAKING IN \\ STUDENTS OF COMPULSORY SECONDARY EDUCATION IN CUENCA
}

\author{
Amparo Martínez Cano** \\ Universidad de Castilla-La Mancha
}

\section{RESUMEN}

Surge esta investigación por la gran preocupación acerca de la cualificación de los trabajadores en las competencias que exige la sociedad actual. La solución está en la preparación de los trabajadores en dichas competencias profesionales a través de programas de orientación y educación para la carrera a lo largo de la vida.

En la Fundamentación Teórica se estudian los avances en la Orientación para la Carrera, los conceptos de Autoconocimiento y de Toma de Decisiones y el Programa "Tu Futuro Profesional", (TFP) de la Doctora Repetto.

La metodología de investigación consiste en la aplicación del Programa "TFP", en alumnos de ESO, de Centros Públicos de la ciudad de Cuenca, durante tres cursos académicos (1999/2000, 2000/2001 y 2001/2002), con el programa estadístico SPSS y la prueba "T" de Student, para comprobar las diferencias en Autoconocimiento y la Toma de decisiones de los alumnos antes y después de la aplicación del programa "TFP", encontrándose diferencias significativas en ambos.

Palabras clave: Auto-conocimiento, desarrollo de la carrera, toma de decisión.

\section{ABSTRACT}

This research stems from the need of skilled workers successfully integrated in the labour market in today's society. The key to meet to this demand consists on instructing workers in the professional performances through continuous career guidance. This instruction should specially be conducted in

\footnotetext{
* Amparo Martínez Cano, Profesora de la Universidad de Castilla-La Mancha, Escuela Universitaria de Magisterio de Cuenca, Departamento de Pedagogía, área de Didáctica y Organización Escolar. Es Doctora en Pedagogía. Sus líneas de investigación son: La Cultura Escolar, el Desarrollo Sostenible en Castilla la Mancha, Metodología ABP y la Educación en el último Siglo en Castila- La Mancha. E-mail:Amparo.Martinez@uclm.es.
} 
young students between 12 and 18 years old. This stage corresponds to secondary school, when a large number of young people go into the labour market.

The theoretic al foundation, considers the latest principles of career guidance as well as the notions of self-knowledge and decision-making, Professor Repetto's program "TFP", "Tu Futuro Profesional".

In the research methodology, the TFP program is tested on students of compulsory secondary education from public schools in Cuenca, throughout three academic years (1999/2000, 2000/2001, 2001/2002), with the help of SPSS programme and Student "T". The students who followed "TFP" proved to have better self-knowledge and decision-making skills. The advantage was significant in both.

Key words: Self-knowledge, career development, decision-making.

\section{Introducción}

El Programa con el que se ha trabajado,"Tu Futuro Profesional" (TFP), (Repetto, 1999) pertenece a la Orientación para el Desarrollo Profesional y se fundamenta en: Teorías Psicopedagógicas del desarrollo de la carrera y de la transición de la escuela a la vida adulta, Teorías Sociológicas del desarrollo de la carrera y de la transición de la escuela a la vida activa y en el Enfoque Constructivista. Es un Modelo comprensivo, integrado en el currículo escolar y a nuestro juicio el más completo para el desarrollo de habilidades y competencias de los estudiantes en su desarrollo profesional. Repetto (1992, 1994, 1996, 2000, 2002 y 2003). Se presenta en tres niveles: (12-14 años), Primer nivel, (14-16 años), Segundo nivel y (16-18 años), Tercer nivel. Se trabajan en dicho programa cuatro Módulos: MÓDULO I. Autoconocimiento. Entrenamiento en las estrategias para lograr el conocimiento de uno mismo y de los demás. Krumboltz (1996), Fierro (1996), Rodríguez Cao (1995); MÓDULO II. Toma de Decisiones. Entrenamiento en las estrategias para dirigir el proyecto de vida. Gelatt (1962), Cruz (1996); MÓDULO III. Exploración para la carrera. Entrenamiento en las estrategias para identificar las competencias y relacionarlas con las características de las ocupaciones. Holland (1985 y 1987); MÓDULO IV. Planificación y Gestión de la carrera. Entrenamiento en las estrategias para diseñar la ocupación y construir el futuro laboral. Romero Rodríguez (2004).

Dentro de cada módulo se especifican: Objetivo general, ambiente, conceptos, clima y los objetivos específicos. Cada Módulo está formado por varias unidades en las que se especifican: Objetivos, conceptos básicos, tamaño del grupo, temporalización, recursos, espacio físico y los pasos de su realización.

A lo largo de los tres cursos de recogida de datos (1999/2000, 2000/2001 y 2001/2002, se aplican los dos primeros Módulos (Autoconocimiento y Toma de decisiones) del Programa "TFP", en alumnos de tres Institutos de Secundaria de Cuenca, de entre 12-14 años. A continuación se especifican los aspectos generales de dichos módulos:

Se entiende por Autoconocimiento el proceso en constante crecimiento e interacción sujeto-medio por el que el alumno va formándose el concepto de sí mismo relacionándolo con las valoraciones que le otorgan los otros. A esa definición la Dra. Repetto llega a través de diferentes posiciones teóricas.

Toma de Decisiones se define como el: "Proceso continuo y secuencial, que necesita de constantes revisiones, según la información que va adquiriendo el sujeto a lo largo del desarrollo de su carrera (Martínez, 2004). 


\section{Método}

Dada la escasez de programas comprensivos e integrados en el currículo que capaciten en el desarrollo de las habilidades y competencias de los estudiantes en su desarrollo profesional, el problema que se pretende conocer es el siguiente: ¿El Programa “TFP” mejora de los niveles de autoconocimiento y toma de decisiones de los alumnos de ESO de Cuenca?, de este problema se derivan las siguientes hipótesis:

- Hipótesis primera: existen diferencias significativas entre los Grupos Experimental y Control, en la muestra elegida, tras la aplicación del Módulo de Autoconocimiento del Programa "TFP".

- Hipótesis segunda: existen diferencias significativas entre el Grupo Experimental y el Grupo de Control, en el Módulo de Toma de Decisiones, en la muestra elegida, tras la aplicación del Programa "TFP".

- Hipótesis tercera: existen diferencias significativas entre las Ganancias del Grupo Experimental y el Grupo Control, en la muestra elegida tras la aplicación del Programa “TFP", en el Módulo de Autoconocimiento.

- Hipótesis cuarta: existen diferencias significativas entre las Ganancias del Grupo Experimental y el Grupo Control, en la muestra elegida, tras la aplicación del Programa "TFP", en el Módulo de Toma de Decisiones.

Las variables que se consideran son el Programa "TFP", cómo independiente y las ganancias alcanzadas por los alumnos después de la aplicación de los módulos de Autoconocimiento y Toma de Decisiones, como dependiente.

El diseño es de tipo cuasi-experimental, con observaciones de los grupos experimental y control antes y después de que el grupo experimental reciba el tratamiento.

\begin{tabular}{|l|c|}
\hline Grupos de Control Pretest-Postest. & $01 \quad \mathrm{O} 3$ \\
\hline Grupos Experimentales Pretest / TFP / Postest. & $\mathrm{O} 2 \times \mathrm{O} 4$ \\
\hline Ganancias de los Grupos Experimentales & $\mathrm{Ge}=\mathrm{O} 4-\mathrm{O} 2$ \\
\hline Ganancias de los Grupos Control & $\mathrm{Gc}=\mathrm{O} 3-\mathrm{O} 1$ \\
\hline
\end{tabular}

\begin{tabular}{|c|c|c|c|c|}
\hline Grupos & selección - asignación & Pretest & V. I. & Postest. \\
\hline $\mathrm{E}$ & Azar & $\mathrm{Si}$ & "TFP" & $\mathrm{Si}$. \\
\hline $\mathrm{C}$ & Azar & $\mathrm{Si}$ & --- & $\mathrm{Si}$ \\
\hline
\end{tabular}

La población considerada durante el curso 1999-2000, en alumnos de Centros Públicos de ESO en Cuenca era de 2.600 alumnos y en el curso 2000-2001, 2.549 alumnos.

En cuanto a la muestra, la misma comprende a 365 alumnos.

De esos 365 alumnos de la muestra, como muestra la tabla 1, de primer curso son el $59,2 \%$. En el primer curso se aplicó el módulo de Autoconocimiento y de segundo curso el $40,8 \%$. En segundo curso se aplicó el módulo de Toma de Decisiones. 
TABLA 1: Muestra según el curso.

\begin{tabular}{|l|l|c|c|c|c|}
\hline \multicolumn{2}{|c|}{} & Frecuencia & Porcentaje & $\begin{array}{c}\text { Porcentaje } \\
\text { válido }\end{array}$ & $\begin{array}{c}\text { Porcentaje } \\
\text { acumulado }\end{array}$ \\
\hline \multirow{3}{*}{ Válidos } & 1 & 216 & 58,7 & 59,2 & 59,2 \\
\cline { 2 - 6 } & 2 & 149 & 40,5 & 40,8 & 100,0 \\
\cline { 2 - 6 } & Total & 365 & 99,2 & 100,0 & \\
\hline Perdidos & Sistema & 3 & 0,8 & & \\
\hline Total & & 386 & 100,0 & & \\
\hline
\end{tabular}

Según la tabla 2, la muestra según el Centro de Estudios dónde se aplicó el programa se distribuye de la siguiente forma: Alumnos del IES Lorenzo Hervás y Panduro, el 17,3\%, alumnos del IES Pedro Mercedes, el 56,7\% y alumnos del IES Alfonso VIII, el 26.

TABLA 2: Muestra según el centro de estudios.

\begin{tabular}{|l|l|c|c|c|c|}
\hline \multicolumn{2}{|c|}{} & Frecuencia & Porcentaje & $\begin{array}{c}\text { Porcentaje } \\
\text { válido }\end{array}$ & $\begin{array}{c}\text { Porcentaje } \\
\text { acumulado }\end{array}$ \\
\hline \multirow{3}{*}{ Válidos } & 1 & 63 & 17,1 & 17,3 & 17,3 \\
\cline { 2 - 6 } & 2 & 207 & 56,3 & 56,7 & 74,0 \\
\cline { 2 - 6 } & 3 & 95 & 25,8 & 26,0 & 100,0 \\
\cline { 2 - 6 } & Total & 365 & 99,2 & 100,0 & \\
\hline Perdidos & Sistema & 3 & 0,8 & & \\
\hline Total & & 368 & 100,0 & & \\
\hline
\end{tabular}

Al Grupo Experimental pertenecen el 63,3\% y al Grupo Control el 36,7\%, como se observa en la tabla 3.

TABLA 3: Muestra según el grupo.

\begin{tabular}{|l|l|c|c|c|c|}
\hline \multicolumn{2}{|c|}{} & Frecuencia & Porcentaje & $\begin{array}{c}\text { Porcentaje } \\
\text { válido }\end{array}$ & $\begin{array}{c}\text { Porcentaje } \\
\text { acumulado }\end{array}$ \\
\hline \multirow{3}{*}{ Válidos } & 1 & 134 & 36,4 & 36,7 & 36,7 \\
\cline { 2 - 6 } & 2 & 231 & 62,8 & 63,3 & 100,0 \\
\cline { 2 - 6 } & Total & 365 & 99,2 & 100,0 & \\
\hline Perdidos & Sistema & 3 & 0,8 & & \\
\hline Total & & 368 & 100,0 & & \\
\hline
\end{tabular}


El Sexo de los alumnos se distribuye de la siguiente manera: Masculino, el 52,1\% y Femenino el 47,9\%, (Tabla 4).

TABLA 4: Muestra según el sexo.

\begin{tabular}{|l|l|c|c|c|c|}
\hline \multicolumn{2}{|c|}{} & Frecuencia & Porcentaje & $\begin{array}{c}\text { Porcentaje } \\
\text { válido }\end{array}$ & $\begin{array}{c}\text { Porcentaje } \\
\text { acumulado }\end{array}$ \\
\hline \multirow{3}{*}{ Válidos } & 1 & 190 & 51,6 & 52,1 & 52,1 \\
\cline { 2 - 6 } & 2 & 175 & 47,6 & 47,9 & 100,0 \\
\cline { 2 - 6 } & Total & $\mathbf{3 6 5}$ & $\mathbf{9 9 , 2}$ & $\mathbf{1 0 0 , 0}$ & \\
\hline Perdidos & Sistema & 3 & 0,8 & & \\
\hline Total & & $\mathbf{3 6 8}$ & $\mathbf{1 0 0 , 0}$ & & \\
\hline
\end{tabular}

Los instrumentos de recogida de datos los ofrece el propio programa al término de cada uno de los módulos, tanto para profesores cómo para alumnos, y que son los siguientes:

- Para los profesores:

1. Hoja de Valoración de cada Unidad para el Profesor.

2. Escala de Viabilidad de la aplicación del programa de educación para la carrera destinada al Profesorado.

3. Hoja de Valoración del Módulo I para el Profesor.

4. Hoja de Valoración del Modulo II para el Profesor.

- Para los alumnos:

5. Hoja de Valoración de cada Unidad para el Alumno.

6. Hoja de Valoración Específica del Módulo I para el Alumno.

7. Hoja de Valoración Específica del Módulo II para el Alumno.

\section{Resultados}

Se realizan los siguientes análisis: Primero los profesores llevan a cabo la valoración de los módulos (Gráficos 1 y 2), la viabilidad del programa (Gráficos 3, 4, 5 y 6) y la valoración de cada una de las unidades (Gráficos 7 y 8). Después los alumnos valoran los distintos aspectos de las unidades (desde el gráfico 9 a la 15) y en último lugar la evaluación de los resultados pretest-postest de los grupos Experimental y Control (Tablas 5 y 6).

\section{Valoración de profesores}

Tanto en el Modulo de Autoconocimento cómo en el de Toma de Decisiones, con una media de 2,5 , todas las valoraciones son positivas, siendo el valor más bajo el otorgado a los recursos y el más alto el puntuado para el valor del programa para la transición a la vida adulta seguido del concedido al valor de la participación de los alumnos. 


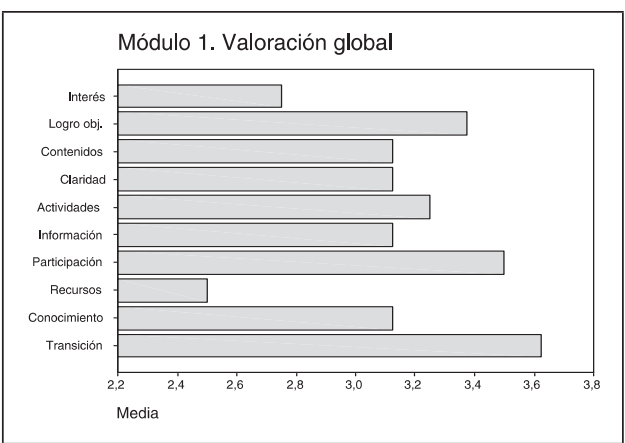

FIGURA 1

Valoración del Módilo I.

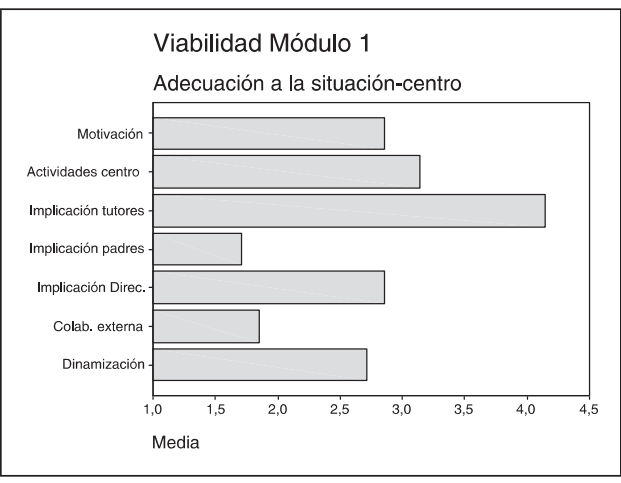

FIGURA 3.

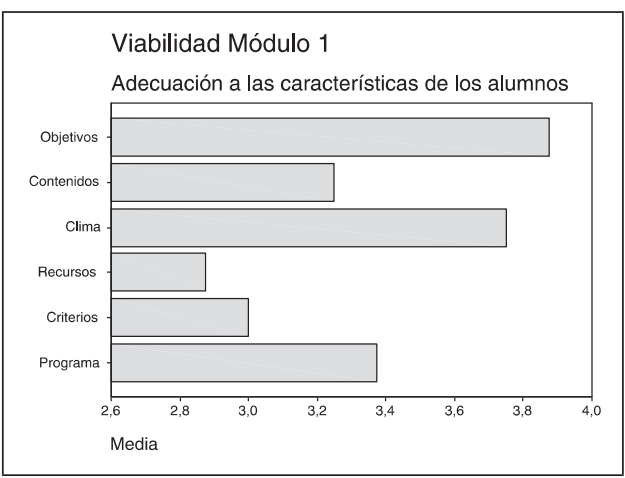

FIGURA 5.

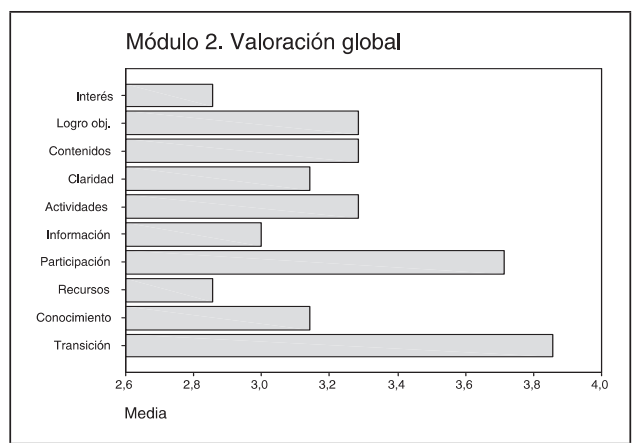

FIGURA 2.

Valoración del Módulo II.

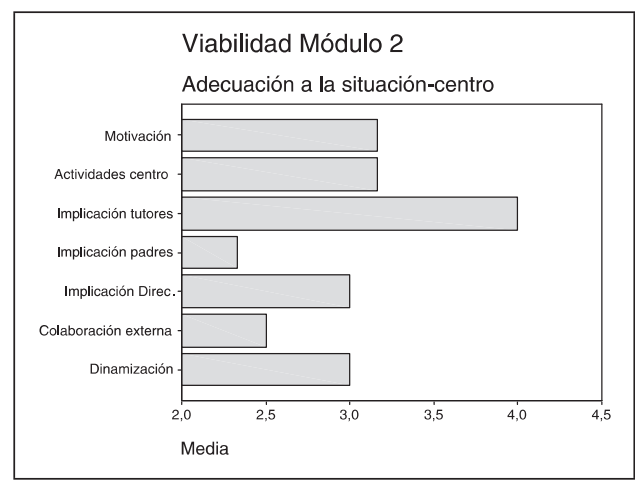

FIGURA 4.

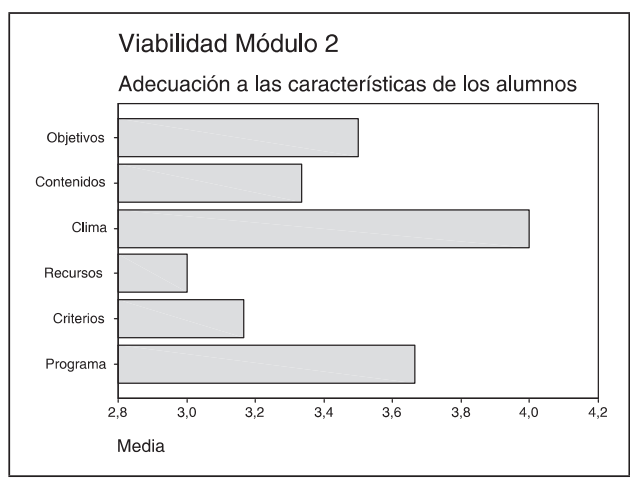

FIGURA 6.

$22($ pp. 17-30) 


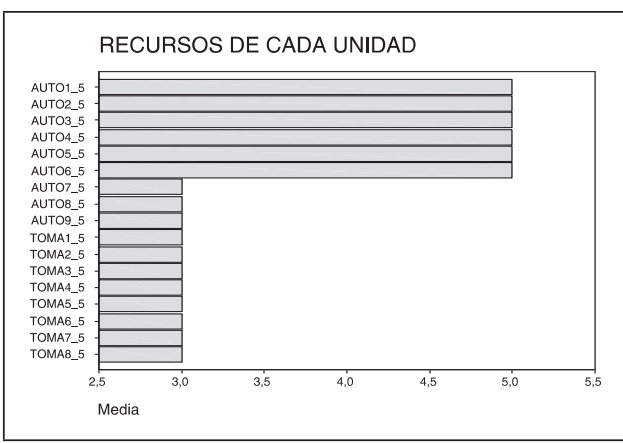

FIGURA 7.

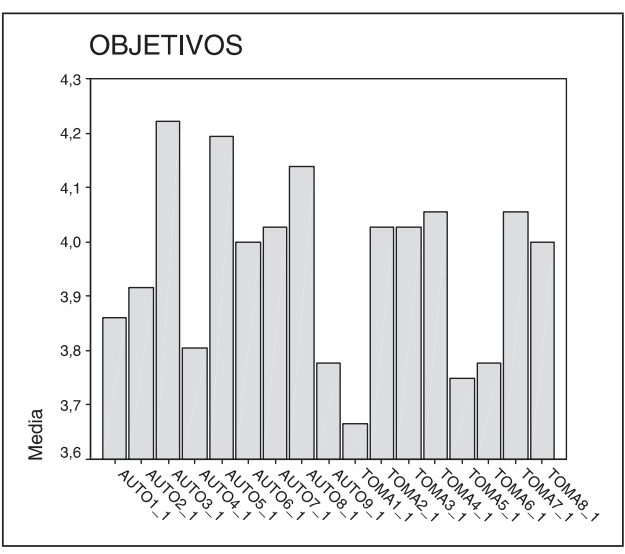

FIGURA 9.

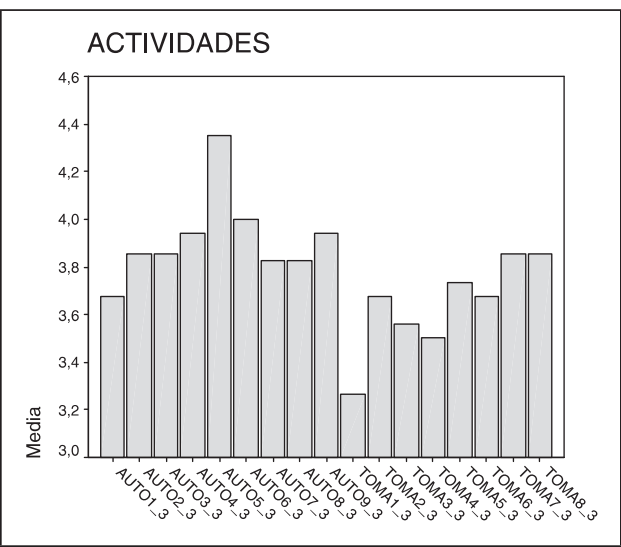

FIGURA 11.

REOP. Vol. 18, $N^{o}$ 1, $2^{e r}$ Semestre, 2007

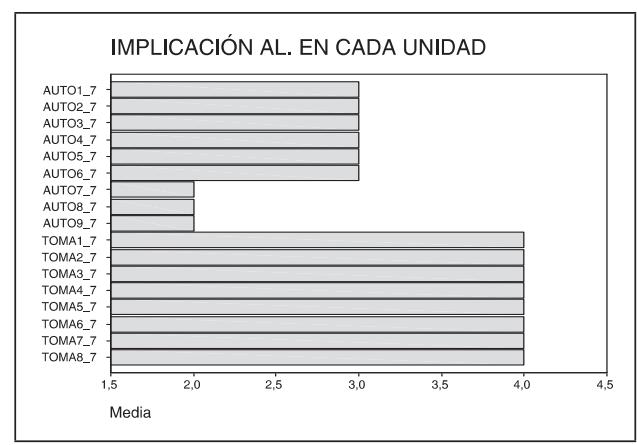

FIGURA 8.

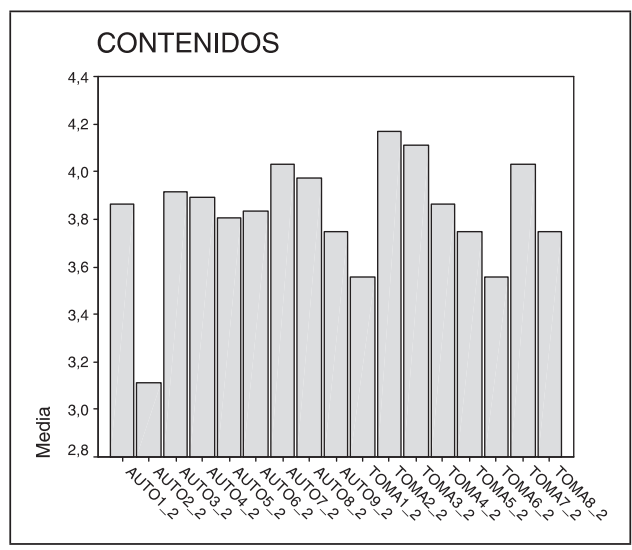

FIGURA 10.

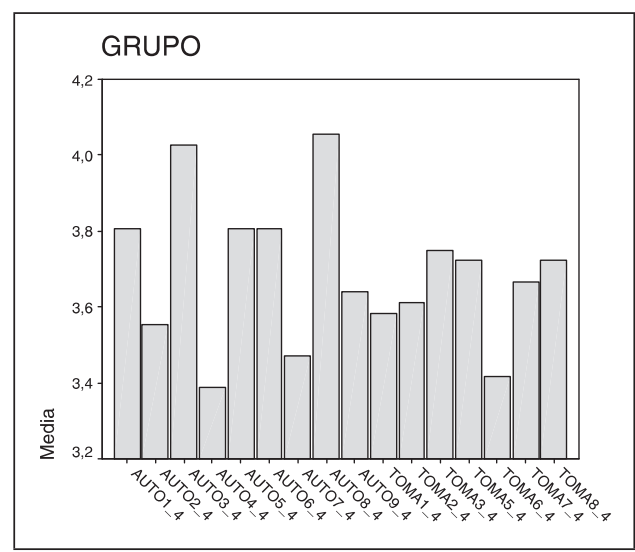

FIGURA 12 


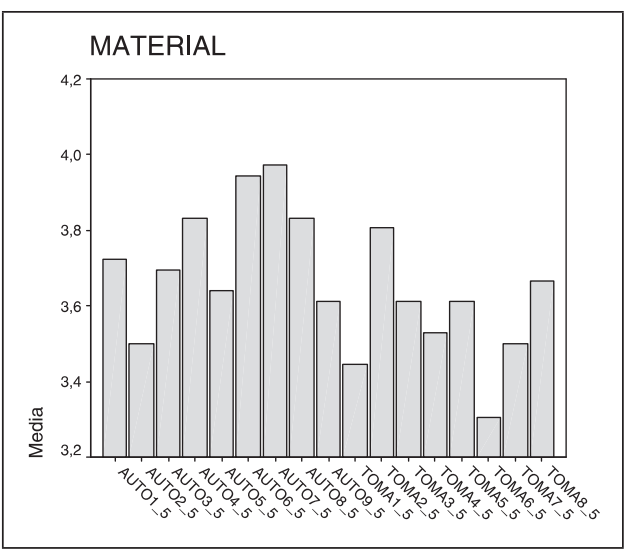

FIGURA 13.

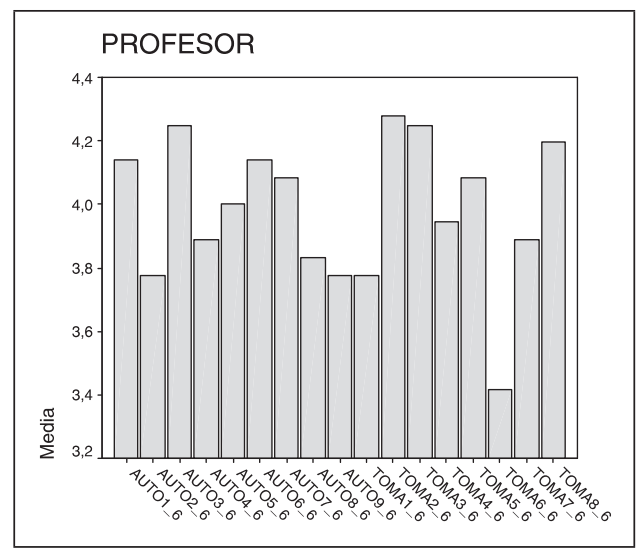

FIGURA 14.

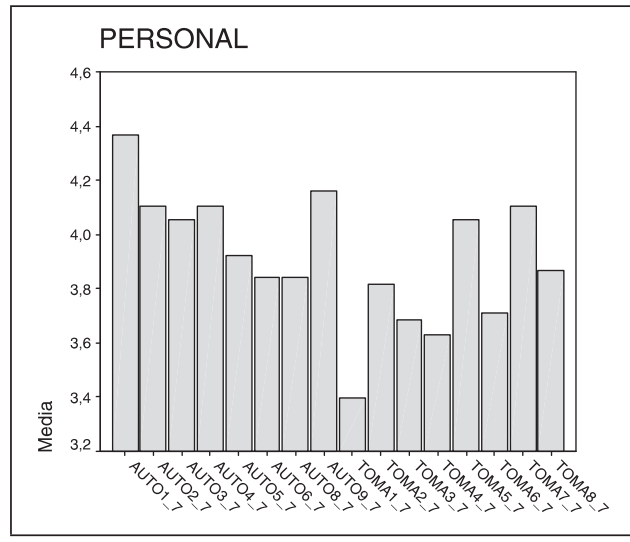

FIGURA 15.

\section{Viabilidad de los Módulos I y II en cuanto a la adecuación a la situación-Centro}

Destacan por encima de la Media las valoraciones de: 4,0, obtenido en Implicación de los tutores y de 3,1, obtenido en Incardinación del programa en las actividades del centro. Por debajo de la Media destacan: El 1,7, de la Implicación de los padres y el 1,9, en Colaboración Externa. Los tutores inciden en señalar la importancia de que padres y medio ambiente se impliquen en las actividades de sus hijos.

\section{Viabilidad de los Módulos I y II en cuanto a la adecuación} a las características de los alumnos

El clima de participación y cohesión es lo más valorado por los docentes del Programa en cuanto a la viabilidad del mismo, en ambos módulos en cuanto a la situación de los alumnos. 


\section{Valoración de las Unidades de los Módulos de Autoconocimiento y de Toma de Decisiones}

Todas las unidades de ambos Módulos se valoran de forma positiva, destacando por lo significativo la Implicación del Alumno cada vez mayor a lo largo del Programa y en el polo opuesto los Recursos que son peor valorados a lo largo del programa pero siempre con puntuaciones por encima del 2,5 de la media.

\section{Valoración de los alumnos}

En segundo lugar tendremos en cuenta las valoraciones los aspectos reflejados en las unidades, según los alumnos. Todos los aspectos evaluados de las Unidades de los dos módulos, obtienen puntuaciones que oscilan entre 3 y 4,4, valores muy altos teniendo en cuenta el 2,5 de la media. Destacan sobre el resto de valoraciones las realizadas sobre: La Implicación del Profesor, la Implicación del Alumno, el Logro de Objetivos y el Funcionamiento del Grupo.

\section{Resultados de la aplicación del Tratamiento}

Para obtener los resultados de la aplicación del tratamiento con pretest y postest, se aplica la prueba T de Student, mediante el programa SPSS, además de la prueba de Levene para igualdad de medias y la prueba de Levene para igualdad de varianza. Los resultados se presentan a continuación (Tablas 5 y 6), y representan las unidades con resultados significativos en cada uno de los Módulos y en las ganancias de los mismos.

Con los resultados anteriores podremos realizar los contrastes de las hipótesis $1^{\mathrm{a}}, 2^{\mathrm{a}}$, $3^{\mathrm{a}}$ y $4^{\mathrm{a}}$ :

\section{Contraste de la hipót esis $1^{a}$}

El Grupo Control del Pretest al Postest, del Módulo de Autoconocimiento, tiene diferencias significativas en las unidades: 4, (Ventajas e inconvenientes de depender) y 9, (Guía de autoevaluación).

El Grupo Experimental desde el Pretest al Postest en el Módulo de Autoconocimiento tiene diferencias significativas en las unidades: 1, (Habilidades Motoras) 2, (Características propias y ajenas) 4, (Ventajas e inconvenientes de depender) y 5, (Entrevista pública). Luego, aceptamos la hipótesis primera.

\section{Contraste de la hipótesis $2^{a}$}

El Grupo Control del Pretest al Postest, del Módulo de Toma de Decisiones, tiene diferencias significativas en la unidad 5, (Cosas aprendidas dentro y fuera de la escuela).

El Grupo Experimental desde el Pretest al Postest en el Módulo de Toma de Decisiones tiene diferencias significativas en las unidades:1, (Razones de elegir) 2, (Actividades que) 
3, (Razones para preferir) 4, (Destrezas de ocio que) 5, (Cosas que aprendiste) 6, (Experiencias divertidas) 7, (Pasos a seguir para aprender) y 8, (Fases de las decisiones). Luego, aceptamos la Hipótesis segunda.

TABLA 5.

\begin{tabular}{|c|c|c|c|}
\hline $\begin{array}{l}\text { Contraste } \\
\text { en Módulo I }\end{array}$ & Programa SPSS & Unidad y significación & $\%$ \\
\hline \multirow{7}{*}{$\begin{array}{l}\text { Pretest GC y } \\
\text { GE }\end{array}$} & \multirow{6}{*}{$\begin{array}{l}\text { Prueba } T \text {, igualdad de } \\
\text { medias }\end{array}$} & 1. Habilidades motoras & 0,000 \\
\hline & & 2. Características propias y ajenas & 0,000 \\
\hline & & 4. Dependencia/Independencia & 0,001 \\
\hline & & 5. Entrevista pública & 0,009 \\
\hline & & 7. Habilidades personales & 0,001 \\
\hline & & 9. Guía de autoevaluación & 0,001 \\
\hline & $\begin{array}{l}\text { Prueba de Leveue, } \\
\text { igualdad de varianzas }\end{array}$ & 4. Dependencia/Independencia & 0,01 \\
\hline \multirow{4}{*}{$\begin{array}{l}\text { Postest GC y } \\
\text { GE }\end{array}$} & \multirow{3}{*}{$\begin{array}{l}\text { Prueba } T \text {, igualdad de } \\
\text { medias }\end{array}$} & 5. Entrevista pública & 0,01 \\
\hline & & 7. Habilidades personales & 0,105 \\
\hline & & 8. Habilidades sociales & 0,141 \\
\hline & $\begin{array}{l}\text { Prueba de Levene, } \\
\text { igualdad de varianzas }\end{array}$ & 1. Habilidades motoras & 0,001 \\
\hline $\begin{array}{l}\text { Pretest- } \\
\text { postest GC }\end{array}$ & $\begin{array}{l}\text { Prueba } T \text {, muestras } \\
\text { relacionadas }\end{array}$ & 4. Dependencia/independencia & 0,025 \\
\hline \multirow{5}{*}{$\begin{array}{l}\text { Pretest- } \\
\text { postest GE }\end{array}$} & \multirow{5}{*}{$\begin{array}{l}\text { Prueba } T \text {, muestras } \\
\text { relacionadas }\end{array}$} & 9. Guía de autoevaluación & 0,013 \\
\hline & & 1. Habilidades motoras & 0,000 \\
\hline & & 2. Características propias y ajenas & 0,000 \\
\hline & & 4. Dependencia/independencia & 0,000 \\
\hline & & 5. Entrevista pública & 0,010 \\
\hline \multirow[t]{7}{*}{ Ganancias } & \multirow{4}{*}{$\begin{array}{l}\text { Prueba Levene, } \\
\text { igualdad de varianzas }\end{array}$} & 3. Actividades que se realizan sólo o en grupo & 0.01 \\
\hline & & 4. Dependencia/independencia & 0,01 \\
\hline & & 5. Entrevista pública & 0.008 \\
\hline & & 7. Habilidades personales & 0,019 \\
\hline & \multirow{3}{*}{$\begin{array}{l}\text { Prueba Levene, } \\
\text { igualdad de medias }\end{array}$} & 2. Características propias y ajenas & 0,018 \\
\hline & & 4. Dependencia/independencia & 0.000 \\
\hline & & 9. Guía de autoevaluación & 0,053 \\
\hline
\end{tabular}


TABLA 6.

\begin{tabular}{|c|c|c|c|}
\hline $\begin{array}{l}\text { Contraste en } \\
\text { Módulo II }\end{array}$ & Programa SPSS & Unidad y significación & $\%$ \\
\hline \multirow[t]{3}{*}{ Pretest CG y GE } & Prueba T, Iguladad de medias & 5. Cosas que aprender & 0,01 \\
\hline & \multirow{2}{*}{$\begin{array}{l}\text { Prueba de Levene, igualdad de } \\
\text { varianzas }\end{array}$} & 7. Pasos para aprender destrezas & 0,05 \\
\hline & & 8. Fases de las decisiones & 0,05 \\
\hline \multirow{6}{*}{$\begin{array}{l}\text { Postest CG y } \\
\text { GE }\end{array}$} & \multirow[t]{4}{*}{ Prueba T, igualdad de medias } & 2. Búsqueda de alternativas & 0,24 \\
\hline & & 3. Razones de preferir & 0,01 \\
\hline & & 7. Pasos para aprender destrezas & 0,24 \\
\hline & & 8. Fases de las decisiones & 0,06 \\
\hline & \multirow{2}{*}{$\begin{array}{l}\text { Prueba de Levene, igualdad de } \\
\text { varianzas }\end{array}$} & 7. Pasos para aprender destrezas & 0,10 \\
\hline & & 8. Fases de las decisiones & 0,01 \\
\hline $\begin{array}{l}\text { Pretest-postest } \\
\text { GC }\end{array}$ & Prueba $\mathrm{T}$, muestras relacionadas & 5. Cosas que aprender & 0,025 \\
\hline \multirow{8}{*}{$\begin{array}{l}\text { Pretest-postest } \\
\text { GE }\end{array}$} & \multirow[t]{8}{*}{ Prueba $\mathrm{T}$, muestras relacionadas } & 1. Razones de elegir & 0,000 \\
\hline & & 2. Búsqueda de alternativas & 0,000 \\
\hline & & 3. Razones de preferir & 0,000 \\
\hline & & 4. Destrezas de ocio & 0,000 \\
\hline & & 5. Cosas que aprender & 0,001 \\
\hline & & 6. Experiencias divertidas & 0,001 \\
\hline & & 7. Pasos para aprender destrezas & 0,000 \\
\hline & & 8. Fases de las decisiones & 0,000 \\
\hline \multirow[t]{13}{*}{ Ganancias } & \multirow{6}{*}{$\begin{array}{l}\text { Prueba Levene, igualdad de } \\
\text { varianzas }\end{array}$} & 1. Razones de elegir & 0,005 \\
\hline & & 2. Búsqueda de alternativas & 0,003 \\
\hline & & 3. Razones de preferir & 0,000 \\
\hline & & 4. Destrezas de ocio & 0,011 \\
\hline & & 5. Cosas que aprender & 0,019 \\
\hline & & 7. Pasos para aprender destrezas & 0,000 \\
\hline & \multirow{7}{*}{$\begin{array}{l}\text { Prueba Levene, igualdad de } \\
\text { medias }\end{array}$} & 1. Razones de elegir & 0,000 \\
\hline & & 2. Búsqueda de alternativas & 0,002 \\
\hline & & 3. Razones de preferir & 0,000 \\
\hline & & 4. Destrezas de ocio & 0,005 \\
\hline & & 5. Cosas que aprender & 0,001 \\
\hline & & 7. Pasos para aprender destrezas & 0,003 \\
\hline & & 8. Fases de las decisiones & 0,015 \\
\hline
\end{tabular}




\section{Contraste de la hipótesis $3^{a}$}

Existen diferencias significativas entre las Ganancias del Grupo Experimental y el Grupo Control, en el Módulo de Autoconocimiento en las unidades: 2, (Características propias y ajenas) 3, (Actividades que realizar) 4, (Ventajas e inconvenientes de depender) y 9, (Guía de autoevaluación). Luego, aceptamos la hipótesis tercera.

\section{Contraste de la hipótesis $4^{a}$}

Existen diferencias significativas en las ganancias del Grupo Experimental y Control, en el módulo de Toma de decisiones en las Unidades: 1, (Razones de elegir) 2, (Actividades que) 3, (Razones para preferir) 4, (Destrezas de ocio que) 5, (Cosas que aprendiste) 7, (Pasos a seguir para aprender) y 8, (Fases de las decisiones). Luego, aceptamos la hipótesis cuarta.

\section{Conclusiones}

De acuerdo con los resultados obtenidos se puede concluir que:

Las diferencias en el Módulo de Autoconocimiento, entre Grupo Experimental y Control, al comienzo de la aplicación del mismo son menores que tras su aplicación.

Las diferencias en el Módulo de Toma de Decisiones entre Grupo Experimental y Control, al comienzo de la aplicación del mismo son menores que tras su aplicación.

El Grupo Experimental cambia en muchas más unidades, significativamente, que el Grupo de Control, en el Módulo de Autoconocimiento tras la aplicación de dicho Módulo.

El Grupo Experimental cambia en mayores aspectos de una manera significativa, que el Grupo de Control en el Módulo de Toma de Decisiones, tras la aplicación de este Módulo.

Hay Ganancias significativas en el Módulo de Autoconocimiento, en la mayor parte de las unidades.

Hay Ganancias significativas en el Módulo de Toma de Decisiones, en la mayor parte de las unidades.

Tanto los alumnos cómo los profesores valoran positivamente el Programa. Los profesores valoran de forma positiva la viabilidad del Programa en cada uno de sus Módulos.

Merece destacar el hecho de que los alumnos evalúan con puntuaciones todavía más altas que los profesores, siendo el Trabajo en Grupo lo mas valorado por profesores y alumnos y los Recursos empleados lo menos valorado por ambos. Los resultados de la aplicación del Pretest y el Postest confirman tanto en el Módulo de Autoconocimiento cómo en el de Toma de decisiones que el "TFP", puede ser un valioso recurso en el incremento del autoconocimiento y la toma de decisiones de los alumnos de Educación Secundaria Obligatoria.

Entre las implicaciones prácticas destaca que el Programa "TFP" debe de estar incardinado en la docencia, Padilla y Rodríguez Dieguez, (1994), mediante infusión curricular, en este sentido puede aplicarse tanto completo cómo cada Módulo por separado. Como se ha observado a lo largo de esta investigación en la que sólo se aplicaron dos de los cuatro mó- 
dulos, los resultados son positivos aunque conforme avanza la aplicación del mismo esos resultados de incrementan.

\section{Referencias bibliográficas}

Anaya, D. y Repetto, E. (1994). "Estudios de la madurez vocacional de los estudiantes de Educación Secundaria mediante el Career Development Inventory", Educación XXI, 1, 159-174.

Cruz, J. M. (1996). “Evaluación del programa ¡Tengo que decidirme!, Revista de Orientación Educativa y Vocacional, $\mathrm{N}^{\mathrm{o}} 11$.

Fierro, A. (1996): El conocimiento de sí mismo. in: Fierro, A. (Comp.), Manual de Psicología de la personalidad. Barcelona: Paidos.

Gelatt, H. B. (1962). "Decision-Making: A conceptual frame of reference for counseling", Journal of Counseling psychology, 9, 3. 240-245.

Holland, J. L. (1985 y 1997). Making vocational choices: A theory of vocational personalities and work environments ( $2^{\mathrm{a}}$ ed.). Englewood Cliffs: Prentice-Hall.

Krumboltz, J. D. (1996). A Learning Theory of Career Counseling, in: Savickas, M \& Walsh, B (eds.), Integrating Career Theory and practice. Palo Alto CA.: Davies - Black/ Press.

Martínez, A. (2004). El desarrollo del Autoconocimiento y de la Toma de decisiones de los alumnos de la Educación Secundaria Obligatoria en la Ley Orgánica de Calidad de la Educación. (Universidad de Castilla - La Mancha), n 197 de la colección de tesis doctorales. IBSN 84-8427-373-3.

Padilla, M. T. y Rodríguez Diéguez (1994). "Educación para la carrera y reforma del Sistema Educativo". Revista de Orientación Educativa y Vocacional, No 7.

Repetto Talavera, E. (1992). "El desarrollo de la carrera a lo largo de la vida y la Orientación de los recursos humanos en las organizaciones". Revista de Orientación Educativa y Vocacional, Año III, No 4.

Repetto Talavera, E. (1996). La orientación profesional y el desarrollo de la carrera en la Enseñanza, in: Sanz Oro. R. (ed.), et al. Tutoría y Orientación. (25-44). Barcelona: Cedecs.

Repetto Talavera, E. (1999). Tu Futuro Profesional. Libro del Profesor Tutor y Libro del Alumno. 3 Vol. Madrid: Cepe.

Repetto Talavera, E. \& Gil, J. A. (2000). "Programa de Orientación, Tu Futuro Profesional y su Evaluación", Material entregado para el Curso de Verano de la UNED, Beneficios educativos, sociales y económicos de la Orientación para la Carrera Profesional. XI Edición. Denia: UNED.

Repetto Talavera, E. (2002). Modelos de Orientación e Intervención Psicopedagógica. Marco conceptual y metodológico, Volumen 1. Madrid: UNED.

Repetto Talavera, E. (2003). Modelos de Orientación e Intervención Psicopedagógica. Intervenciones Psicopedagógicas para el desarrollo del aprendizaje, de la carrera y de la persona. Volumen 2. Madrid: UNED. 
Rodriguez Cao, L. (1995). “Aproximación al autoconcepto". Revista de Orientación Educativa y Vocacional, Año VI, No 9.

Romero Rodríguez, S. (2004). "Aprender a construir Proyectos profesionales vitales" Revista de Orientación y Psicopedagogía, Vol. 15, № 2, $2^{\circ}$ semestre, 337-354. ISSN: 11397853.

Padilla, M. T. y Rodríguez Diéguez (1994). "Educación para la carrera y reforma del Sistema Educativo". Revista de Orientación Educativa y Vocacional, N ${ }^{\mathrm{o}} 7$.

Fecha de recepción: 26-07-05

Fecha de revisión: 21-06-06

Fecha de aceptación: 08-05-07 